\title{
Baylisascaris procyonis un nemátodo del mapache urbano, agente zoonótico emergente por considerar como diagnóstico diferencial de larva migrans en Costa Rica. Revisión.
}

\section{Baylisascaris procyonis, a nematode in urban raccoons and emerging zoonotic agent to be con- sidered as a differential diagnosis of larva migrans in Costa Rica. Review.}

\section{Mario Baldia1,b1 $\mathbb{}$, Martha Piche ${ }^{\mathrm{a} 1}$, Mario Romero ${ }^{\mathrm{a} 1}$, Sabine Elisabeth Hutter ${ }^{\mathrm{b} 1}$, Víctor} Montenegro ${ }^{\mathrm{a} 2}$, Chris Walzer ${ }^{\mathrm{b} 2}$.

a. ${ }^{1}$ Programa de Investigación en Enfermedades Tropicales y ${ }^{2}$ Laboratorio de Parasitología, Escuela de Medicina Veterinaria. Universidad Nacional Heredia. Costa Rica. Email: $\$ mario.baldi.salas@una.cr, mmpo88@gmail.com, luisma903@gmail.com,victor.montenegro.hidalgo@una.cr

b. $\quad{ }^{1}$ Instituto de Salud Pública. ${ }^{2}$ Instituto de Ecología y Vida Silvestre. Universidad Veterinaria de Viena, Austria. Email: ‘baldim@medvetuni.ac.at, sabine.hutter@vetmeduni.ac.at, walzerc@staff.vetmeduni.ac.at

Recibido: 10 de noviembre de 2016. Corregido: 7 de marzo de 2017. Aceptado: 17 de marzo de 2017.

Resumen: El ascaridio Baylisascaris procyonis es un agente parasitario de importancia para la salud pública, siendo su huésped definitivo el mapache común (Procyon lotor). Un nuevo informe alerta sobre la presencia de estas lombrices en poblaciones de mapaches en la zona urbana de Costa Rica, y hace énfasis en la necesidad de conocer más sobre este parásito, su ciclo de vida y cómo prevenir su transmisión en humanos y animales domésticos. Esta comunicación es una breve revisión sobre los aspectos más importantes de este nematodo y sus efectos negativos en la salud animal y pública. Además, se hace hincapié en los mejores métodos de diagnóstico etiológico y los más apropiados para animales domésticos, así como el tratamiento y la prevención en perros. El presente artículo pretende formar conciencia en los trabajadores de la salud pública y animal sobre la necesidad de tomar el Bayliascaris procyonis dentro de su diagnóstico diferencial de larvas migrantes en Costa Rica.

Palabras Clave: Mapaches, Baylisascaris procyonis, Salud Pública, Salud Animal, Larva migrans.

Abstract: The zoonotic helminth Baylisacaris procyonis is a parasite in urban raccoons (Procyon lotor) of increasing public health concern. A new report alerts about the presence of these roundworms in urban raccoon populations in Costa Rica stressing the need to understand the parasite, its life cycle and preventive measures to avoid transmission to humans and pets. This is a brief review of the most remarkable features of this parasite and its negative effects on animal and public health. Additionally, emphasis is given on the best and most appropriate etiologic diagnostic methods in domestic animals, as well as treatment and prevention in dogs. This article is meant to raise awareness in public and animal health caretakers to consider Bayliascaris procyonis as differential diagnosis for larva migrans in Costa Rica.

Keywords: Raccoons, Baylisascaris procyonis, Public Heath, Animal Health, Larva migrans. 


\section{Introducción}

El reciente reporte sobre la presencia del nemátodo Baylisascaris procyonis en poblaciones de mapaches urbanos en Costa Rica por (Baldi et al. 2016), señala el riesgo zoonótico asociado a la interfaz humano y animales silvestres. El B. procyonis es un nemátodo propio de los mapaches o mapachines (Procyon lotor). Este nematodo, reportado en poblaciones de mapaches del área metropolitana de Costa Rica, con una prevalencia de un $50 \%$ de un total de 20 animales analizados. El parásito se clasifica como un agente responsable de causar lesiones en órganos internos por larvas migrantes en vertebrados, los cuales actúan como hospedadores incidentales (incluyendo el ser humano) (Sorvillo et al. 2002). La presencia de este nemátodo en ambientes tropicales es una alerta para médicos veterinarios, médicos de humanos y los responsables de la salud pública nacional sobre conocer esta zoonosis para lograr su prevención de manera efectiva.

\section{El parásito}

El Baylisascaris procyonis es un nemátodo que pertenece a la superfamilia Ascaridoidea, siendo su huésped definitivo el mapache común. Esta especie tiene una larga historia evolutiva con su hospedador, por lo cual, los efectos perniciosos sobre el mapache son menores (Kazacos 2001). Además, de B. procyonis, existen ocho especies más dentro del género Baylisacaris spp, el cual incluye el $B$. columnaris siendo su huésped definitivo las mofetas (Mephitis macroura); la especie B. potosís, recientemente descrita para las martillas (Potos flavus), todos ellos con potencial zoonótico (Kazacos 2001; Tokiwa, et al. 2014). Para estas dos especies, sus hospederos definitivitos se encuentran distribuidos a lo largo de Mesoamérica.

El B. procyonis tiene un ciclo de vida de tipo directo (Figura 1). Los parásitos adultos alcanzan un tamaño de 15-20 centímetros en hembras y 10-15 centímetros en machos, en el intestino delgado del mapache (Roussere et al. 2003; Anderson \& Chabaud 2009). Las hembras eliminan huevos al ambiente en las heces, los cuales, bajo condiciones de temperatura ambiental $\left(18-24^{\circ} \mathrm{C}\right)$ y una humedad relativa $(80-100 \%)$, desarrollarán el estadio de latencia de huevos larvados $\left(L_{1}\right)$. Después de, aproximadamente, dos semanas son capaces de alcanzar el estadio infectante $\left(L_{2}\right)$ (Page et al. 1999; Kazacos 2001) En este estadio es ingerido, en primera instancia, por mapaches jóvenes de sustratos, letrinas, madrigueras o al consumir alimentos contaminados. Los huevos son muy resistentes a las condiciones ambientales y pueden sobrevivir, incluso, por años (Shafir et al. 2011).

Alternativamente, el huevo larvado puede, también, ser ingerido por huéspedes paraténicos, especialmente pequeños mamíferos y aves que se alimentan de material orgánico no digerido, presentes en las letrinas (lugares comunes de defecación de mapaches) o de alimentos contaminados con huevos (Figura 2). En el nuevo hospedero, la larva atraviesa la pared intestinal y se traslada, vía hemática, alcanzando los pulmones, hígado, ojos y sistema nerviosos central donde las larvas son enquistadas (Hirsch et al. 2014). Los vertebrados, que sufren de larva migrante de tipo nerviosa, presentan cambios de comportamiento asociado encefalitis eosinófilica a nivel central. Por ello, terminan muriendo por las lesiones 
causadas o siendo presa fácil de los mapaches (Fitzgerald et al. 1991; Kazacos 2001). Por ambos rutas, el nemátodo logra completar su ciclo de vida en su hospedador definitivo.

\section{Interacción mapache-mascotas}

Recientemente, la presencia de mapaches en el Gran Área Metropolitana va en aumento, al igual que los reportes anecdóticos en hoteles de playa de las zonas costeras del país. El fenómeno de sinantropía, en mapaches (competencia de plantas y animales silvestres para habitar en ecosistemas antropizados), puede estar asociada a varios factores, entre ellos la plasticidad de comportamiento de la especie para adaptarse a condiciones nuevas, disponibilidad de alimento en los asentamientos humanos (desechos orgánicos), así como a un desarrollo urbanístico sin planificación, condiciones presentes en Costa Rica e ideales para esta especie (Conover 2001; Baldi et al. 2016).

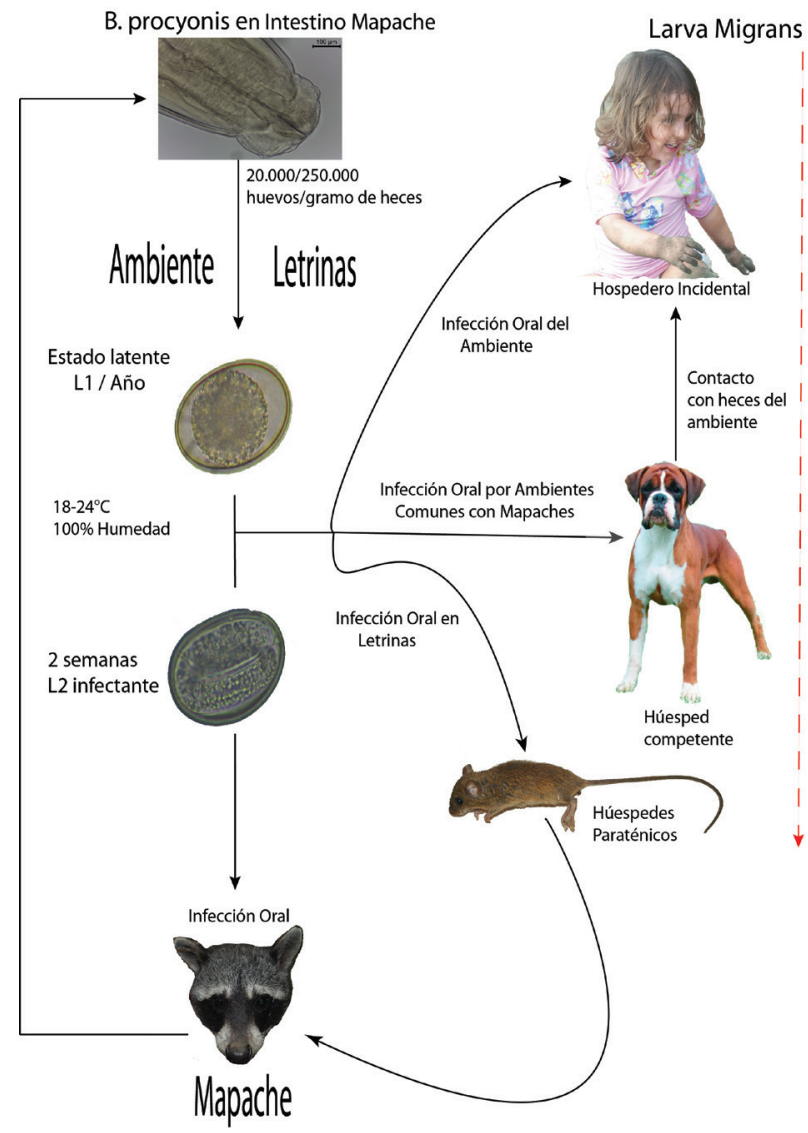

Figura 1. Ciclo de vida del Baylisacaris procyonis en su hospedero definitivo (Procyon lotor) y sus hospederos incidentales. 


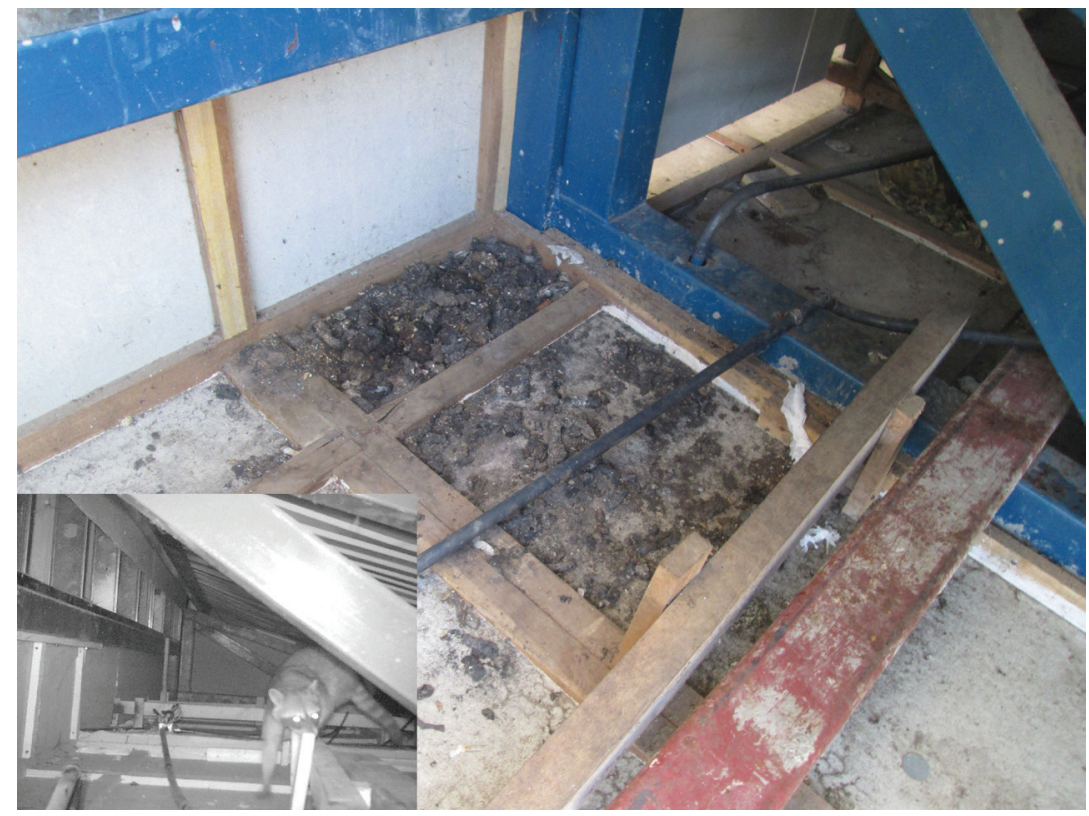

Figura 2. Letrina en cielorrasos, usadas por mapaches como áreas comunes de defecación.

La presencia aumentada de mapaches en el Área Metropolitana costarricense se traduce en un conflicto. El cual puede ir desde el uso de cielorrasos como áreas de letrinas, hasta la incursión a cocinas y áreas de alimentación en las casas invadidas (Navares 2014). Uno de las interacciones más notable es la que ocurre entre los mapaches, humanos y/o sus mascotas (perros y gatos). Estas interacciones pueden estar asociadas con la capacidad de transmisión de este nemátodo a animales domésticos y a seres humanos (Roscoe 1993; Bowman et al. 2005; Graeff-Teixeira et al 2016). Bowman 2000, reportó el Baylisacaris procyonis en cachorros afectados con larva migrans, presentaron cuadros de tipo nervioso, observándose movimiento en círculo, parálisis del tren posterior, letargia, ceguera, ataxia y postración (Bowman 2000). Adicionalmente, los perros pueden actuar como hospedero "adecuado", porque se han identificado perros adultos capaces de eliminar huevos de esta especie de manera efectiva. Kazacos (2006), logró demostrar la co-infección de Toxocara canis y Baylisascaris procyonis en un perro de dos años de edad, ilustrando el papel del perro como hospedero efectivo y su rol de diseminador de huevos por su comportamiento de defecación. Además de complicar la identificación del B. procyonis en vivo, en especial en perros coinfectados.

\section{Diagnóstico usado para reconocer el B. procyonis en mascotas}

El principal problema con el B. procyonis y otras especies de este género, es la dificultad de diferenciar sus huevos por métodos estándar como: el examen directo de heces o técnicas 
de flotación (Sheather), al ser confundido con otras especies de ascaridios. El tamaño de los huevos de $B$. procyonis (68 a $76 \times 55$ a $61 \mu \mathrm{m}$ ) y su estructura externa (Figura 3a), lo hace indiferenciable de otros Basylisascaris sp y difícil su diferenciación de otros géneros de Toxocara sin un análisis detallado de la estructura externa del huevo bajo microscopia electrónica (Kazacos \& Turek 1982). La identificación molecular, de secuencias de ADN en huevos o tejido del parásito, es el único método de diagnóstico etiológico efectivo para este género, dado su alta sensibilidad y especificidad (Dangoudoubiyam et al. 2009). En caso de enviar los perros a necropsia, el material de la larva, localizado en nódulos parasitarios o en tejidos enquistados o la misma larva, localizadas en el intestino delgado, se les deberá realizar la identificación molecular por medio de la técnica de reacción en cadena de la polimerasa (PCR) de punto final (Figura 3b) o en tiempo real. Esta prueba, por su alta sensibilidad y especificidad, es el método de elección para clasificar al nemátodo (Franssen et al. 2013).

\section{Tratamiento en perros y medidas de prevención.}

El rol del perro: como víctima, huésped y diseminador del parásito, debe ser reducida o eliminada en lo posible. Para tal fin es necesario realizar exámenes coprológicos regularmente y desparasitar los perros siguiendo los protocolos de desparasitación para parásitos gastrointestinales.

El uso de desparasitantes con acción antihelmítica son los indicados. Milbemicina Oxima (Interceptorm ${ }^{\circledR}$, Program Plus ${ }^{\circledR}$ y Milbemax ${ }^{\circledR}$ de Novarits, y Trifexis $^{\circledR}$ de Elanco) en dósis de $0.5-0.9 \mathrm{mg} / \mathrm{kg}$ vía oral, luego un examen coprológico de rutina. Esta dosis debe repetirse un mes después para asegurar la eliminación total de los nemátodos (Bowman et al. 2005). Adicionalmente, estos principios activos, en combinación con otros antiparasitarios de amplio espectro, son recomendados (Pamoato de Pirantel, Fenbendazole, Flubendazole, Albendazole, Piperazina, dosis altas de Ivermectina o Moxidectina, o combinaciones de Pirantel, Febantel, Praziquantel e Ivermectinas) en las dosis orales establecidas (Kazacos 2006). Se recomienda el uso de vapor de agua y flameo de superficies en ambientes contaminados, como método de limpieza, dado que los huevos de $B$. procyonis posee susceptibilidad a altas temperaturas (mayores a $60^{\circ} \mathrm{C}$ ). La incineración de estructuras de madera afectada es lo adecuado para la eliminación total de los huevos. Se recomiendo agregar agua hirviendo sobre las superficies con material fecal $y$, posteriormente, aplicar cloro comercial sin diluir (3-5\% Hipoclorito de Sodio) o el uso de Kileno-etanol como desinfectantes en ellas, dejándolos actuar, al menos por una hora, una vez retirado el material fecal (Kazacos 2001; Shafir et al. 2011).

Dadas las altas prevalencias de huevos de ascaridios en suelos de áreas públicas nacionales (parques infantiles y áreas de recreo) (Paquet-Durand et al. 2007), así como las prevalencias reportadas de ascaridios en cachorros de perros en tiendas de mascotas (Calderón et al. 2008), sumado a la reciente descripción y presencia de $B$. procyonis en mapaches en el Valle Central (Baldi et al. 2016), se debe resaltar la necesidad de establecer medidas preventivas y profilácticos enfocadas hacia los perros de familia. 


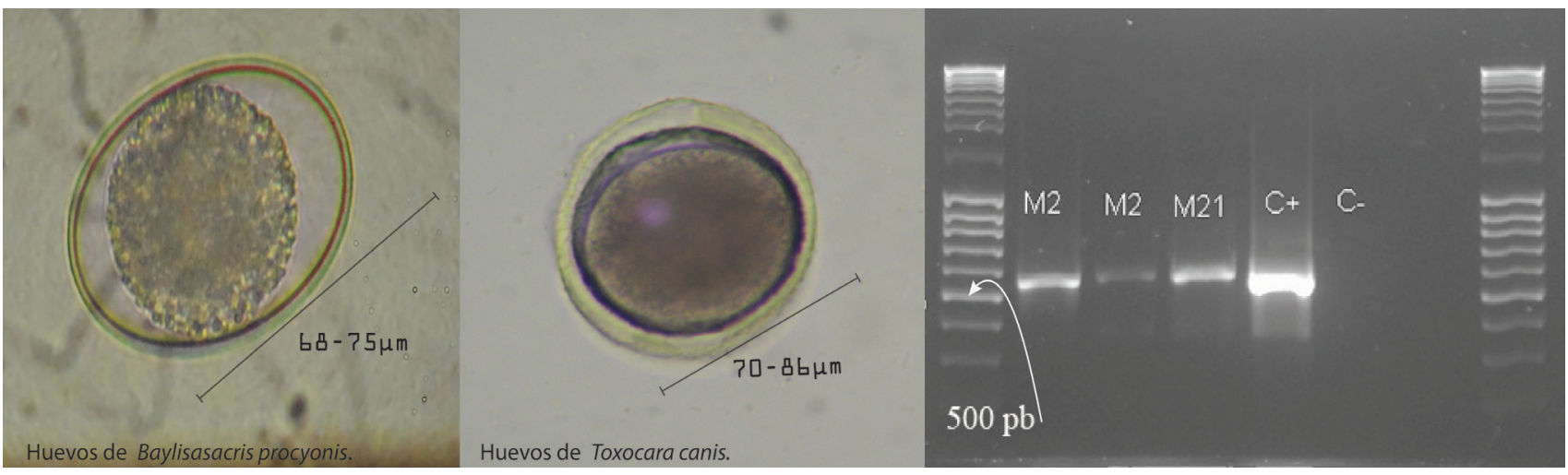

Figura 3: a: Tamaño y estructura del huevo de Baylisascaris procyonis y Toxocara canis. b: Muestras fecales (M2, M21) de mapaches positivas a Baylisascaris procyonis por medio de la prueba de PCR.

\section{Larva migrans en humanos}

Los seres humanos actúan como huéspedes incidentales del Baylisascaris procyonis, sufriendo la misma patología de larva migrans que otros vertebrados. Los humanos se infectan al ingerir huevos larvados de $B$. procyonis cuando, accidentalmente, consumen alimento, tierra o beben agua contaminada o ponen objetos contaminados en la boca (Gavin et al. 2005; Graeff-Teixeira et al. 2016). Las presentaciones de larva migrans son las mismas reportadas para Toxocara spp; definidas como Larva migrans viceral (LMV), Larva migrans ocular (LMO) y Larva migrans neural (LMN). Esta última, especialmente agresiva en personas afectadas (Saffra et al. 2010). La contaminación del ambiente se da, en primera instancia, por heces infectadas en letrinas o áreas circundantes (Bauer 2013).

Los casos de LMN, asociados a B. procyonis, documentados en los Estados Unidos, corresponden a pacientes inmunocomprometidos o que presentan algún nivel de pica (geofagia) y niños, en especial niños menores de 3 años, siendo esta la población más susceptible, por su naturaleza de poner objetos en la boca (Park et al. 2000; Wise et al. 2005). De igual manera, personas asociadas a contactos directos o indirectos con mapaches (por ejemplo profesionales de la vida silvestre), podrían adquirir el parásito si no se toma las medidas de prevención adecuadas como: uso de guantes, lavado de manos posterior a la manipulación de mapaches o sus heces.

\section{Tratamiento en humanos}

Desafortunadamente, el tratamiento de larva migrans en humanos es limitado, una vez que el parásito logra ingresar al sistema nervioso central o periférico (Murray \& Kazacos 2004). Las opciones para el tratamiento se centran en el uso de antiparasitarios, capaces de cruzar la barrera hematoencefálica. Se recomienda, en estos casos, los tratamientos antihelminticos con principios activos con base en albendazoles, vía oral por un mes (Peters 
et al. 2012).Estos antiparasitarios, en combinación con corticosteroides, son el tratamiento de elección para reducir la inflamación al nivel central (Wise et al. 2005). Sin embargo, los casos de LMN tienen un mal pronóstico.

\section{Conclusiones}

Bajo la luz de este nuevo reporte, médicos veterinarios y encargados de la salud pública deben tomar el Baylisascaris procyonsis dentro de los diagnósticos diferenciales de larva migrans, e implementar medidas de prevención enfocadas en los animales domésticos, en especial perros. Estos, como diseminadores potenciales de huevos de B. procyonis dentro de la epídemiología de esta zoonosis. Por ello, es fundamental conocer y usar los métodos diagnósticos y tratamientos adecuados para prevenir esta parasitosis en perros y humanos susceptibles.

Agradecimiento Jorge Hernández y Katherine Sánchez Paniagua por las fotografías, al Dr. Norbeto Baldi, por sus valiosos comentarios al manuscrito. Fondo Institucional de Desarrollo Académico-2013-Universidad Nacional- Universidad de Costa Rica, Fondo del SistemaConsejo Nacional de Rectores (ACUERDO-VI-167-2013).

\section{Referencias}

Anderson, R.C., Chabaud, A.G. \& Willmott, S.: Keys to the Nematode Parasites of Vertebrates. Archival Volume. CAB International, Wallingford (UK); 2009: 463. ISBN-13: 978-184593-572-6.

Baldi, M., Alvarado, G., Smith, S., Santoro, M., Bolaños, N., Jiménez, C., Hutter, S. E., \& Walzer, C. 2016. Baylisascaris procyonis Parasites in Raccoons, Costa Rica, 2014. Emerging Infectious Diseases. 22(8):1502-1503. doi:10.3201/eid2208.151627.

Bauer C. 2013. Baylisascariosis-Infections of animals and humans with 'unusual' roundworms. Veterinary Parasitology. Volume 193(4): 404-412. doi./10.1016/j. vetpar.2012.12.036.

Bowman, D.D. 2000. Baylisascaris Procyonis in Dogs. In: Companion and Exotic Animal Parasitology, Bowman D.D. (Ed.). International Veterinary Information Service. http:// www.ivis.org/advances/Parasit_Bowman/bowman_baylisascariasis/ivis.pdf

Bowman, D.D., Ulrich, M.A., Gregory, D.E., Neumann, N.R., Legg, W. \& Stansfield, D. 2005. Treatment of Baylisascaris procyonis infections in dogs with milbemycin oxime. Parasitology 129(3-4): 285-90. doi./10.1016/j.vetpar.2004.02.030.

Calderón, S., De Oliveira, J., Hernández, J., Jiménez, M. \& Muñoz, P. 2008. Parásitos gastrointestinales en caninos menores de seis meses comercializados en tiendas de mascotas de la Gran Área Metropolitana de Costa Rica. Ciencias Veterinarias 26(1): 21-35. Disponible en: http://www.revistas.una.ac.cr/index.php/veterinaria/article/ view/4682 
Conover, M. R. Resolving human-wildlife conflicts. 2001. The science of wildlife damage management / Michael Conover. Boca Raton, Fla.: Lewis Publishers. ISBN: 978-156670-538-7.

Dangoudoubiyam, S., Ramesh V. \& Kazacos, K.R. 2009. PCR Assays for Detection of Baylisascaris Procyonis Eggs and Larvae. The Journal of Parasitology. 95(3): 571-77. doi: 10.1645/GE-1905.1.

Fitzgerald, S. D., White, M.R. \& Kazacos, K.R. 1991. Encephalitis in Two Porcupines Due to Baylisascaris Larval Migration. Journal of Veterinary Diagnostic Investigation 3(4): 359-62. doi:10.1177/104063879100300421.

Franssen, F., Xie, K., Sprong, H. \& van der Giessen, J. 2013. Molecular Analysis of Baylisascaris Columnaris Revealed Mitochondrial and Nuclear Polymorphisms. Parasites \& Vectors 6: 124. doi: 10.1186/1756-3305-6-124.

Gavin, P.J., Kazacos, K.R., Shulman, S.T. \& Gavin, T.E. 2005. Baylisascariasis. Clinical Microbiology Reviews. 18(4): 703-18. doi: 10.1128/CMR.18.4.703-718.2005.

Graeff-Teixeira, C., Morassutti, L. \& Kazacos, K.R. 2016. Update on Baylisascariasis, a Highly Pathogenic Zoonotic Infection. Clinical Microbiology Reviews 29(2). doi: 10.1128/ CMR.00044-15.

Hirsch, B.T., Prange, S., Hauver, S. \& Gehrt, S. D. 2014. Patterns of Latrine Use by Raccoons (Procyon Lotor) and Implication for Baylisascaris Procyonis Transmission. Journal of Wildlife Diseases. 50(2): 243-49. doi: 10.7589/2013-09-251.

Kazacos, K.R. \& Turek, J.J. 1982. Scanning Electron Microscopy of the Labia of Baylisascaris Procyonis (Nematoda). The Journal of parasitology 68(4): 634-41. doi: DOI: 10.2307/3280921

Kazacos, K.R. 2001. Baylisascaris Procyonis and Related Species. In Parasitic Diseases of Wild Mammals, WM Samuel, MJ Pybus, and AA Kocan (Eds.). lowa State University Press, Ames, lowa (2001): 301-341. ISBN-081382978X.

Kazacos, K. R. 2006. Unusual Fecal Parasite in a Dog. applied cytology: 3-5. Disponible en: http://www.cliniciansbrief.com/column/applied-cytology/unusual-fecal-parasitedog.

Murray, W.J.; Kazacos, K.R. 2004. Raccoon roundworm encephalitis. In Clinical infectious diseases: an official publication of the Infectious Diseases Society of America 39 (10), pp. 1484-1492. doi: 10.1086/425364.

Navares, V. 2014. Distribución Potencial de la Ocurrencia del Conflicto Ser Humano-Mapache Norteño (Procyon Lotor) y la Percepción de los Pobladores Afectados de La Gran Área Metropolitana de Costa Rica. Tesis. Universidad Nacional de Costa Rica.

Page, L.K., Swihart, R. K. \& Kazacos, K.R. 1999. Implications of Raccoon Latrines in the Epizootiology of Baylisascariasis. Journal of Wildlife Diseases 35(3): 474-80. doi: 


\subsection{9/0090-3558-35.3.474.}

Paquet-Durand, I. Hernández, J., Dolz, G., Romero, J.J., Schbieder, T., Epe, C. 2007. Prevalence of Toxocara Spp., Toxascaris Leonina and Ancylostomidae in Public Parks and Beaches in Different Climate Zones of Costa Rica. Acta Tropica 104(1): 30-37. doi: 10.1016/j. actatropica.2007.06.011

Park, S. Y., Glaser, C., Murray, W.J., Kazacos, K.R., Rowley, H.A., Frederick, D.R., Bass, N. 2000. Raccoon Roundworm (Baylisascaris Procyonis) Encephalitis: Case Report and Field Investigation. Pediatrics 106 (4) e1-5. http://pediatrics.aappublications.org/ content/106/4/e56.long.

Peters J.M., Madhavan V.L., Kazacos K.R., Husson R.N., Dangoudoubiyam S., Soul J.S. 2012. Good outcome with early empiric treatment of neural larva migrans due to Baylisascar is procyonis. Pediatrics. 129(3): e806-11. doi: 10.1542/peds.2011-2078.

Roscoe, E. 1993. Epizootiology new of canine raccoons distemper in New Jersey racoons. Journal of Wildlife Diseases 29(3):390-395. http://www.jwildlifedis.org/toc/ jwdi/29/3.

Roussere, G. P., Murray, W.J., Raudenbush, C.B., Kutilek, M.J., Levee, D.J., Kazacos, K. R. 2003. Raccoon Roundworm Eggs near Homes and Risk for Larva Migrans Disease, California Communities. Emerging infectious diseases 9(12): 1516-22. doi: 10.3201/ eid0912.030039

Saffra, N. A. Perlman, J.E., Desai, R.U., Kazacos, K.R., Coyle, C.M., Machado, F.S., Kedhar, S.R., Engelbert, M. \& Tanowitz, H.B. 2010. Baylisascaris Procyonis Induced Diffuse Unilateral Subacute Neuroretinitis in New York City. Journal of Neuroparasitology 1: 1-4. doi. $10.4303 / \mathrm{jnp} / \mathrm{N} 100401$.

Shafir, S.C, Sorvillo, F.J., Sorvillo, T. \& Eberhard, M.L. 2011. Viability of Baylisascaris Procyonis Eggs. Emerging Infectious Diseases 17(7): 1293-95. doi: 10.3201/eid1707.101774.

Sorvillo, F., Ash, L.R., Berlin, O. G. W. \& Morse, S. A. 2002. Baylisascaris Procyonis: An Emerging Helminthic Zoonosis. Emerging Infectious Diseases 8(4): 355-59. doi: 10.3201/eid0804.010273.

Tokiwa, T., Nakamura, S., Taira, K., Yumi, U. 2014. Baylisascaris Potosis N. Sp., a New Ascarid Nematode Isolated from Captive Kinkajou, Potos Flavus, from the Cooperative Republic of Guyana. Parasitology International (63): 591-96. doi: 10.1016/j.parint.2014.03.003.

Wise, M.E., Sorvillo, F.J., Shafir, S.C., Lawrence, R.A., Berlin, O.G. 2005 Severe and Fatal Central Nervous System Disease in Humans Caused by Baylisascaris Procyonis, the Common Roundworm of Raccoons: A Review of Current Literature. Microbes and Infection 7(2): 316-22. doi: 10.1016/j.micinf.2004.12.005 